Acta Crystallographica Section E

Structure Reports

Online

ISSN 1600-5368

\section{(2E)-3-(2-Bromophenyl)-1-(4,4"-difluoro- $5^{\prime}$-methoxy-1, $1^{\prime}: 3^{\prime}, 1^{\prime \prime}$-terphenyl-4'-yl)- prop-2-en-1-one}

\section{Hoong-Kun Fun, ${ }^{a *} \mp$ Tze Shyang Chia, ${ }^{a}$ S. Samshuddin, B. Narayana ${ }^{b}$ and B. K. Sarojinic}

${ }^{a}$ X-ray Crystallography Unit, School of Physics, Universiti Sains Malaysia, 11800 USM, Penang, Malaysia, ${ }^{\mathbf{b}}$ Department of Studies in Chemistry, Mangalore University, Mangalagangotri 574 199, India, and ' Department of Chemistry, P. A. College of Engineering, Nadupadavu, Mangalore 574 153, India

Correspondence e-mail: hkfun@usm.my

Received 26 March 2012; accepted 30 March 2012

Key indicators: single-crystal X-ray study; $T=100 \mathrm{~K}$; mean $\sigma(\mathrm{C}-\mathrm{C})=0.006 \AA$; $R$ factor $=0.071 ; w R$ factor $=0.134 ;$ data-to-parameter ratio $=21.5$.

In the title compound, $\mathrm{C}_{28} \mathrm{H}_{19} \mathrm{BrF}_{2} \mathrm{O}_{2}$, the central benzene ring makes dihedral angles of 62.51 (18), 46.23 (18) and $48.19(18)^{\circ}$ with the bromo-substituted benzene ring and two terminal fluoro-substituted benzene rings, respectively. In the crystal, molecules are linked by $\mathrm{C}-\mathrm{H} \cdots \mathrm{F}$ hydrogen bonds into infinite chains along [110]. Weak $\mathrm{C}-\mathrm{H} \cdots \pi$ and $\pi-\pi$ interactions [centroid-centroid distance $=3.683$ (2) $\AA$ ] also occur and short intermolecular F...F contacts [2.833 (4) $\AA$ ] are observed.

\section{Related literature}

For related structures and background to terphenyl chalcones, see: Fun et al. (2011a,b, 2012). For reference bond lengths, see: Allen et al. (1987). For the stability of the temperature controller used in the data collection, see: Cosier \& Glazer (1986).<smiles>COc1cc(-c2ccc(F)cc2)cc(-c2ccc(F)cc2)c1C(=O)/C=C/c1ccccc1Br</smiles>

\section{Experimental}

Crystal data

$\mathrm{C}_{28} \mathrm{H}_{19} \mathrm{BrF}_{2} \mathrm{O}_{2}$

$M_{r}=505.34$

Monoclinic, $C 2 / c$

$a=22.4861$ (6) §

$b=6.9006(2) \AA$

$c=28.6933(8) \AA$

$\beta=101.286(2)^{\circ}$

\section{Data collection}

Bruker SMART APEXII CCD diffractometer

Absorption correction: multi-scan (SADABS; Bruker, 2009)

$T_{\min }=0.533, T_{\max }=0.863$

Refinement

$R\left[F^{2}>2 \sigma\left(F^{2}\right)\right]=0.071$

$w R\left(F^{2}\right)=0.134$

$S=1.11$

6414 reflections

$V=4366.2(2) \AA^{3}$

$Z=8$

Mo $K \alpha$ radiation

$\mu=1.92 \mathrm{~mm}^{-1}$

$T=100 \mathrm{~K}$

$0.37 \times 0.12 \times 0.08 \mathrm{~mm}$

24190 measured reflections 6414 independent reflections 4483 reflections with $I>2 \sigma(I)$ $R_{\text {int }}=0.076$

\section{Table 1}

Hydrogen-bond geometry $\left(\AA,{ }^{\circ}\right)$.

$C g 1$ and $C g 2$ are the centroids of $\mathrm{C} 1-\mathrm{C} 6$ and $\mathrm{C} 10-\mathrm{C} 15$ rings, respectively.

\begin{tabular}{lllll}
\hline$D-\mathrm{H} \cdots A$ & $D-\mathrm{H}$ & $\mathrm{H} \cdots A$ & $D \cdots A$ & $D-\mathrm{H} \cdots A$ \\
\hline $\mathrm{C} 28-\mathrm{H} 28 A \cdots \mathrm{F} 2^{\mathrm{i}}$ & 0.96 & 2.51 & $3.448(4)$ & 166 \\
$\mathrm{C} 4-\mathrm{H} 4 A \cdots C g 1^{\mathrm{ii}}$ & 0.93 & 2.99 & $3.712(5)$ & 136 \\
$\mathrm{C} 20-\mathrm{H} 20 A \cdots C g 2^{\mathrm{iii}}$ & 0.93 & 2.72 & $3.383(4)$ & 129 \\
$\mathrm{C} 27-\mathrm{H} 27 A \cdots C g 1^{\text {iv }}$ & 0.93 & 2.95 & $3.735(4)$ & 143 \\
$\mathrm{C} 28-\mathrm{H} 28 B \cdots C g 2^{\mathrm{v}}$ & 0.96 & 2.82 & $3.485(4)$ & 128 \\
\hline Symmetry codes: $\quad(\mathrm{i}) \quad x+\frac{1}{2}, y-\frac{1}{2}, z ;$ & (ii) $x,-y-1, z-\frac{1}{2} ; \quad$ (iii) $x, y+1, z ;$ (iv) \\
$-x-1,-y,-z ;(\mathrm{v}) x+\frac{1}{2}, y+\frac{3}{2}, z$. & &
\end{tabular}

Data collection: APEX2 (Bruker, 2009); cell refinement: SAINT (Bruker, 2009); data reduction: $S A I N T$; $\operatorname{program}(\mathrm{s})$ used to solve structure: SHELXTL (Sheldrick, 2008); program(s) used to refine structure: SHELXTL; molecular graphics: SHELXTL; software used to prepare material for publication: SHELXTL and PLATON (Spek, 2009).

HKF and TSC thank Universiti Sains Malaysia (USM) for the Research University Grant (1001/PFIZIK/811160). TSC also thanks the Malaysian Government and USM for the award of a research fellowship. BN thanks the UGC for financial assistance through the SAP and BSR one-time grant for the purchase of chemicals. SS thanks Mangalore University for the research facilities.

Supplementary data and figures for this paper are available from the IUCr electronic archives (Reference: HB6704).

\section{References}

Allen, F. H., Kennard, O., Watson, D. G., Brammer, L., Orpen, A. G. \& Taylor, R. (1987). J. Chem. Soc. Perkin Trans. 2, pp. S1-19.

Bruker (2009). SADABS, APEX2 and SAINT. Bruker AXS Inc., Madison, Wisconsin, USA.

Cosier, J. \& Glazer, A. M. (1986). J. Appl. Cryst. 19, 105-107.

Fun, H.-K., Hemamalini, M., Samshuddin, S., Narayana, B. \& Sarojini, B. K. (2011b). Acta Cryst. E67, o3327-03328. 


\section{organic compounds}

Fun, H.-K., Hemamalini, M., Samshuddin, S., Narayana, B. \& Sarojini, B. K. (2012). Acta Cryst. E68, o163.

Fun, H.-K., Shahani, T., Samshuddin, S., Narayana, B. \& Sarojini, B. K. (2011a). Acta Cryst. E67, o3514.
Sheldrick, G. M. (2008). Acta Cryst. A64, 112-122.

Spek, A. L. (2009). Acta Cryst. D65, 148-155. 


\section{supporting information}

Acta Cryst. (2012). E68, o1314-o1315 [doi:10.1107/S1600536812013852]

\section{(2E)-3-(2-Bromophenyl)-1-(4,4"'-difluoro-5'-methoxy-1,1':3',1'-terphenyl-4'- yl)prop-2-en-1-one}

\section{Hoong-Kun Fun, Tze Shyang Chia, S. Samshuddin, B. Narayana and B. K. Sarojini}

\section{S1. Comment}

In continuation of our work on the synthesis and structures of terphenyl chalcones (Fun et al., 2011a,b), the title compound (I) is now described. The starting material of the title compound was prepared from 4,4'-difluoro chalcone by several steps (Fun et al., 2012).

In the title compound (Fig. 1), the central benzene ring (C10-C15) makes dihedral angles of 62.51 (18), 46.23 (18) and $48.19(18)^{\circ}$ with the bromo-substituted benzene ring (C1-C6) and two terminal fluoro-substituted benzene rings (C16C21 \& C22-C27), respectively. Bond lengths (Allen et al., 1987) and angles are within normal ranges and are comparable to related structures (Fun et al., 2011a,b, 2012).

In the crystal (Fig. 2), molecules are linked by $\mathrm{C} 28-\mathrm{H} 28 \mathrm{~A} \cdots \mathrm{F} 2$ hydrogen bonds into infinite chains along [110]. The crystal is further stabilized by $\mathrm{C}-\mathrm{H} \cdots \pi$ interactions (Table 1), involving $C g 1$ and $C g 2$ which are the centroids of $\mathrm{C} 1-\mathrm{C} 6$ and $\mathrm{C} 10-\mathrm{C} 15$ rings, respectively. $\pi-\pi$ interaction is also observed with $C g 4 \cdots C g 4$ distance of 3.683 (2) $\AA$ [symmetry code: $-1 / 2-X, 3 / 2-Y,-Z]$, where $C g 4$ is the centroid of $\mathrm{C} 22-\mathrm{C} 27$ ring.

\section{S2. Experimental}

To a mixture of 1-(4,4"'-difluoro-5'-methoxy-1,1':3',1"-terphenyl-4'-yl) ethanone (0.338 g, $0.001 \mathrm{~mol})$ and 2-bromobenzaldehyde $(0.185 \mathrm{~g}, 0.001 \mathrm{~mol})$ in $30 \mathrm{ml}$ e thanol, $0.5 \mathrm{ml}$ of $10 \%$ sodium hydroxide solution was added and stirred at $5-10{ }^{\circ} \mathrm{C}$ for $3 \mathrm{~h}$. The precipitate formed was collected by filtration and purified by recrystallization from ethanol. Colourless needles were grown from DMF solution by slow evaporation method and yield of the compound was $79 \%$. (m.p.: $440 \mathrm{~K})$.

\section{S3. Refinement}

All $\mathrm{H}$ atoms were positioned geometrically $[\mathrm{C}-\mathrm{H}=0.93$ and $0.96 \AA]$ and refined using a riding model with $U_{\text {iso }}(\mathrm{H})=1.2$ or $1.5 U_{\mathrm{eq}}(\mathrm{C})$. A rotating group model was applied to the methyl group. 


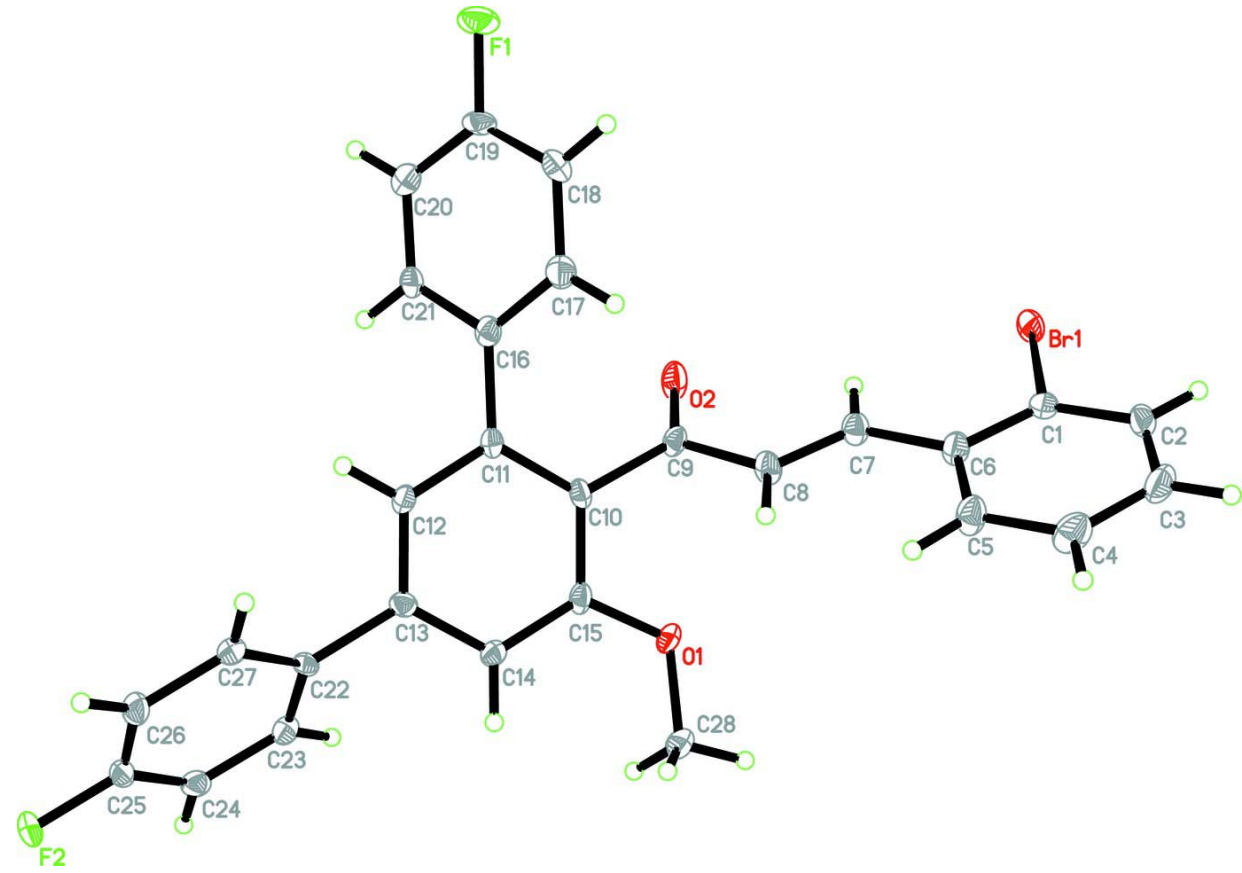

\section{Figure 1}

The molecular structure of the title compound with $50 \%$ probability displacement ellipsoids. 


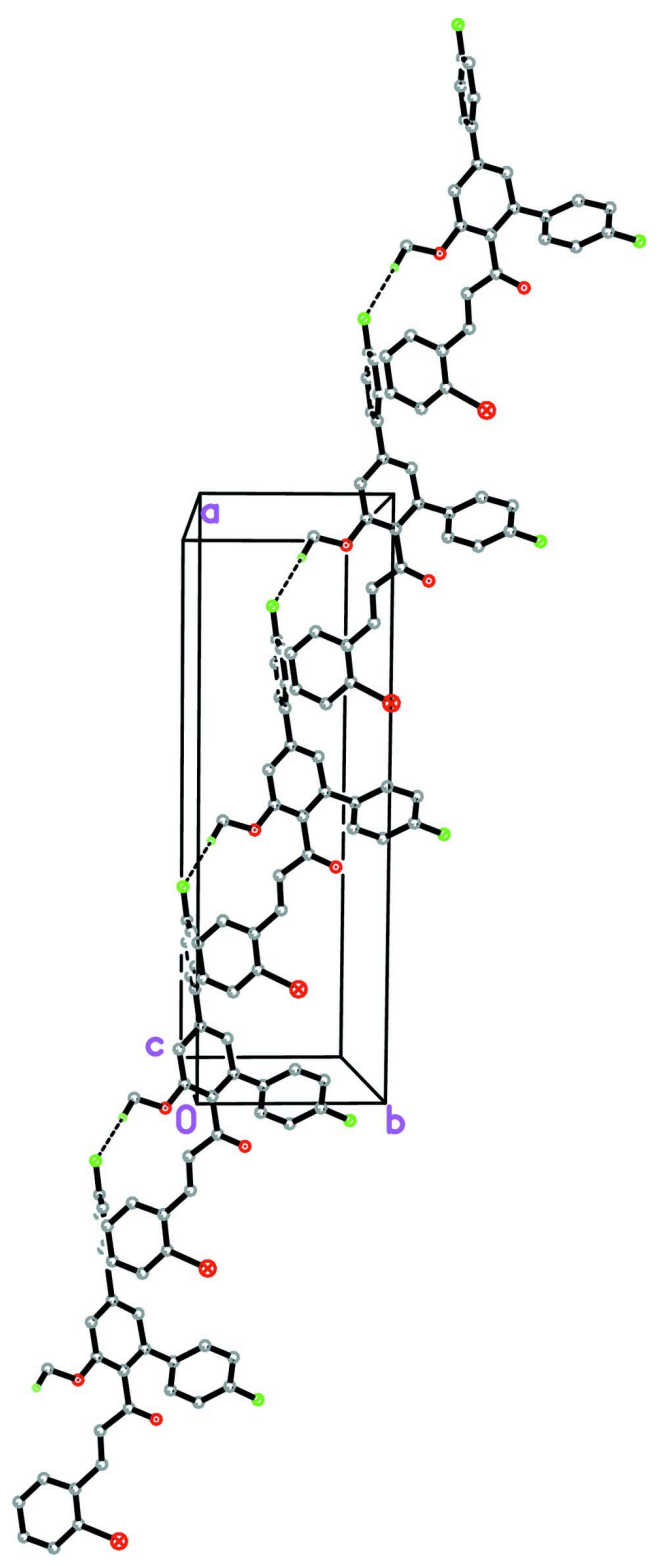

Figure 2

The crystal packing of the title compound. The dashed lines represent the hydrogen bonds.

(2E)-3-(2-Bromophenyl)-1-(4,4'-difluoro-5'-methoxy-1, 1':3',1'-- terphenyl-4'-yl)prop-2-en-1-one

Crystal data

$\mathrm{C}_{28} \mathrm{H}_{19} \mathrm{BrF}_{2} \mathrm{O}_{2}$

$M_{r}=505.34$

Monoclinic, $C 2 / c$

Hall symbol: $-\mathrm{C} 2 \mathrm{yc}$

$a=22.4861(6) \AA$

$b=6.9006(2) \AA$

$c=28.6933(8) \AA$

$\beta=101.286(2)^{\circ}$

$V=4366.2(2) \AA^{3}$

$F(000)=2048$

$D_{\mathrm{x}}=1.538 \mathrm{Mg} \mathrm{m}^{-3}$

Mo $K \alpha$ radiation, $\lambda=0.71073 \AA$

Cell parameters from 4738 reflections

$\theta=2.6-29.4^{\circ}$

$\mu=1.92 \mathrm{~mm}^{-1}$

$T=100 \mathrm{~K}$

Needle, colourless

$Z=8$

$0.37 \times 0.12 \times 0.08 \mathrm{~mm}$ 


\section{Data collection}

Bruker SMART APEXII CCD

diffractometer

Radiation source: fine-focus sealed tube

Graphite monochromator

$\varphi$ and $\omega$ scans

Absorption correction: multi-scan

(SADABS; Bruker, 2009)

$T_{\min }=0.533, T_{\max }=0.863$

\section{Refinement}

Refinement on $F^{2}$

Least-squares matrix: full

$R\left[F^{2}>2 \sigma\left(F^{2}\right)\right]=0.071$

$w R\left(F^{2}\right)=0.134$

$S=1.11$

6414 reflections

299 parameters

0 restraints

Primary atom site location: structure-invariant direct methods
24190 measured reflections

6414 independent reflections

4483 reflections with $I>2 \sigma(I)$

$R_{\text {int }}=0.076$

$\theta_{\max }=30.3^{\circ}, \theta_{\min }=1.5^{\circ}$

$h=-31 \rightarrow 23$

$k=-9 \rightarrow 9$

$l=-40 \rightarrow 39$

Secondary atom site location: difference Fourier map

Hydrogen site location: inferred from neighbouring sites

$\mathrm{H}$-atom parameters constrained

$w=1 /\left[\sigma^{2}\left(F_{\mathrm{o}}{ }^{2}\right)+(0.0321 P)^{2}+25.8799 P\right]$

where $P=\left(F_{\mathrm{o}}^{2}+2 F_{\mathrm{c}}^{2}\right) / 3$

$(\Delta / \sigma)_{\max }<0.001$

$\Delta \rho_{\max }=0.72$ e $\AA^{-3}$

$\Delta \rho_{\min }=-1.09$ e $\AA^{-3}$

Special details

Experimental. The crystal was placed in the cold stream of an Oxford Cryosystems Cobra open-flow nitrogen cryostat (Cosier \& Glazer, 1986) operating at 100.0 (1) K.

Geometry. All e.s.d.'s (except the e.s.d. in the dihedral angle between two 1.s. planes) are estimated using the full covariance matrix. The cell e.s.d.'s are taken into account individually in the estimation of e.s.d.'s in distances, angles and torsion angles; correlations between e.s.d.'s in cell parameters are only used when they are defined by crystal symmetry. An approximate (isotropic) treatment of cell e.s.d.'s is used for estimating e.s.d.'s involving 1.s. planes.

Refinement. Refinement of $F^{2}$ against ALL reflections. The weighted $R$-factor $w R$ and goodness of fit $S$ are based on $F^{2}$, conventional $R$-factors $R$ are based on $F$, with $F$ set to zero for negative $F^{2}$. The threshold expression of $F^{2}>\sigma\left(F^{2}\right)$ is used only for calculating $R$-factors(gt) etc. and is not relevant to the choice of reflections for refinement. $R$-factors based on $F^{2}$ are statistically about twice as large as those based on $F$, and $R$ - factors based on ALL data will be even larger.

Fractional atomic coordinates and isotropic or equivalent isotropic displacement parameters $\left(\AA^{2}\right)$

\begin{tabular}{lllll}
\hline & $x$ & $y$ & $z$ & $U_{\text {iso }} * / U_{\text {eq }}$ \\
\hline F1 & $0.05500(11)$ & $1.3787(4)$ & $0.23294(8)$ & $0.0264(6)$ \\
F2 & $-0.34855(10)$ & $0.4660(3)$ & $-0.01133(9)$ & $0.0242(5)$ \\
Br1 & $0.327887(17)$ & $0.59947(6)$ & $0.161236(15)$ & $0.02040(11)$ \\
O1 & $0.04875(11)$ & $0.3643(4)$ & $0.05277(10)$ & $0.0186(6)$ \\
O2 & $0.11379(12)$ & $0.8145(4)$ & $0.10429(10)$ & $0.0227(6)$ \\
C1 & $0.29189(17)$ & $0.3696(6)$ & $0.17939(13)$ & $0.0164(8)$ \\
C2 & $0.32983(18)$ & $0.2283(6)$ & $0.20296(13)$ & $0.0205(9)$ \\
H2A & 0.3716 & 0.2470 & 0.2094 & $0.025^{*}$ \\
C3 & $0.30549(19)$ & $0.0591(6)$ & $0.21700(15)$ & $0.0235(9)$ \\
H3A & 0.3308 & -0.0355 & 0.2333 & $0.028^{*}$ \\
C4 & $0.2435(2)$ & $0.0309(6)$ & $0.20675(16)$ & $0.0252(10)$ \\
H4A & 0.2271 & -0.0833 & 0.2160 & $0.030^{*}$ \\
C5 & $0.20594(18)$ & $0.1712(6)$ & $0.18288(15)$ & $0.0217(9)$ \\
H5A & 0.1644 & 0.1484 & 0.1755 & $0.026^{*}$
\end{tabular}




$\begin{array}{lllll}\text { C6 } & 0.22854(17) & 0.3485(6) & 0.16924(14) & 0.0175(8) \\ \text { C7 } & 0.18826(17) & 0.5015(6) & 0.14592(14) & 0.0171(8) \\ \text { H7A } & 0.2064 & 0.6160 & 0.1389 & 0.020^{*} \\ \text { C8 } & 0.12818(16) & 0.4921(6) & 0.13392(14) & 0.0162(8) \\ \text { H8A } & 0.1087 & 0.3784 & 0.1398 & 0.019^{*} \\ \text { C9 } & 0.09102(16) & 0.6582(6) & 0.11128(14) & 0.0152(8) \\ \text { C10 } & 0.02379(15) & 0.6264(5) & 0.09697(13) & 0.0134(7) \\ \text { C11 } & -0.01873(16) & 0.7463(5) & 0.11261(13) & 0.0132(7) \\ \text { C12 } & -0.08046(16) & 0.7099(5) & 0.09609(13) & 0.0131(7) \\ \text { H12A } & -0.1089 & 0.7873 & 0.1068 & 0.016^{*} \\ \text { C13 } & -0.10049(16) & 0.5610(5) & 0.06407(13) & 0.0130(7) \\ \text { C14 } & -0.05778(16) & 0.4412(5) & 0.04891(13) & 0.0140(8) \\ \text { H14A } & -0.0705 & 0.3409 & 0.0277 & 0.017^{*} \\ \text { C15 } & 0.00371(16) & 0.4725(6) & 0.06567(14) & 0.0149(8) \\ \text { C16 } & -0.00013(15) & 0.9085(6) & 0.14622(13) & 0.0149(7) \\ \text { C17 } & 0.04441(17) & 0.8892(6) & 0.18743(14) & 0.0195(8) \\ \text { H17A } & 0.0624 & 0.7691 & 0.1952 & 0.023^{*} \\ \text { C18 } & 0.06198(17) & 1.0457(6) & 0.21673(14) & 0.0183(8) \\ \text { H18A } & 0.0915 & 1.0315 & 0.2442 & 0.022^{*} \\ \text { C19 } & 0.03536(17) & 1.2232(6) & 0.20488(14) & 0.0168(8) \\ \text { C20 } & -0.01063(17) & 1.2479(6) & 0.16598(14) & 0.0170(8) \\ \text { H20A } & -0.0298 & 1.3671 & 0.1596 & 0.020^{*} \\ \text { C21 } & -0.02756(15) & 1.0898(6) & 0.13661(13) & 0.0146(7) \\ \text { H21A } & -0.0580 & 1.1046 & 0.1098 & 0.018^{*} \\ \text { C22 } & -0.16673(16) & 0.5323(5) & 0.04452(13) & 0.0129(7) \\ \text { C23 } & -0.18668(17) & 0.5112(6) & -0.00430(14) & 0.0167(8) \\ \text { H23A } & -0.1588 & 0.5115 & -0.0243 & 0.020^{*} \\ \text { C24 } & -0.24795(17) & 0.4900(5) & -0.02318(14) & 0.0172(8) \\ \text { H24A } & -0.2616 & 0.4772 & -0.0558 & 0.021^{*} \\ \text { C25 } & -0.28826(17) & 0.4882(6) & 0.00758(15) & 0.0176(8) \\ \text { C26 } & -0.27040(17) & 0.5068(6) & 0.05564(15) & 0.0182(8) \\ \text { H26A } & -0.2986 & 0.5034 & 0.0754 & 0.022^{*} \\ \text { C27 } & -0.20866(17) & 0.5311(5) & 0.07448(14) & 0.0150(8) \\ \text { H27A } & -0.1955 & 0.5466 & 0.1071 & 0.018^{*} \\ \text { C28 } & 0.03253(18) & 0.1869(6) & 0.02761(15) & 0.0206(9) \\ \text { H28A } & 0.0686 & 0.1224 & 0.0226 & 0.031^{*} \\ \text { H28B } & 0.0068 & 0.2145 & -0.0025 & 0.031^{*} \\ \text { H28C } & 0.0113 & 0.1049 & 0.0459 & 0.031^{*}\end{array}$

Atomic displacement parameters $\left(\AA^{2}\right)$

\begin{tabular}{lllllll}
\hline & $U^{11}$ & $U^{22}$ & $U^{33}$ & $U^{12}$ & $U^{13}$ & $U^{23}$ \\
\hline F1 & $0.0268(13)$ & $0.0204(13)$ & $0.0315(14)$ & $-0.0099(11)$ & $0.0043(10)$ & $-0.0100(11)$ \\
F2 & $0.0090(10)$ & $0.0241(13)$ & $0.0374(14)$ & $0.0011(9)$ & $-0.0004(10)$ & $-0.0055(11)$ \\
Br1 & $0.00986(16)$ & $0.0217(2)$ & $0.0293(2)$ & $-0.00161(18)$ & $0.00283(13)$ & $-0.00163(19)$ \\
O1 & $0.0135(12)$ & $0.0155(14)$ & $0.0275(15)$ & $0.0042(11)$ & $0.0056(11)$ & $-0.0053(11)$ \\
O2 & $0.0125(13)$ & $0.0180(15)$ & $0.0377(18)$ & $0.0013(12)$ & $0.0054(12)$ & $0.0066(13)$ \\
C1 & $0.0171(18)$ & $0.017(2)$ & $0.0142(18)$ & $0.0018(15)$ & $0.0005(14)$ & $-0.0030(15)$
\end{tabular}


supporting information

\begin{tabular}{lllllll} 
C2 & $0.0183(19)$ & $0.026(2)$ & $0.015(2)$ & $0.0079(17)$ & $-0.0029(15)$ & $-0.0044(17)$ \\
C3 & $0.028(2)$ & $0.019(2)$ & $0.023(2)$ & $0.0101(18)$ & $0.0023(17)$ & $0.0017(17)$ \\
C4 & $0.028(2)$ & $0.016(2)$ & $0.033(3)$ & $0.0005(18)$ & $0.0101(19)$ & $0.0042(18)$ \\
C5 & $0.0141(18)$ & $0.019(2)$ & $0.033(2)$ & $0.0015(16)$ & $0.0076(17)$ & $0.0045(18)$ \\
C6 & $0.0157(18)$ & $0.016(2)$ & $0.020(2)$ & $0.0028(15)$ & $0.0029(15)$ & $0.0022(15)$ \\
C7 & $0.0131(17)$ & $0.018(2)$ & $0.021(2)$ & $0.0006(16)$ & $0.0046(15)$ & $0.0023(16)$ \\
C8 & $0.0104(17)$ & $0.018(2)$ & $0.022(2)$ & $-0.0023(15)$ & $0.0057(15)$ & $0.0029(16)$ \\
C9 & $0.0111(17)$ & $0.0147(19)$ & $0.022(2)$ & $0.0018(14)$ & $0.0080(15)$ & $0.0003(15)$ \\
C10 & $0.0082(15)$ & $0.0132(19)$ & $0.0184(19)$ & $-0.0001(14)$ & $0.0017(13)$ & $0.0036(15)$ \\
C11 & $0.0102(16)$ & $0.0144(19)$ & $0.0146(18)$ & $0.0022(14)$ & $0.0013(14)$ & $0.0022(15)$ \\
C12 & $0.0115(16)$ & $0.0111(18)$ & $0.0176(19)$ & $0.0017(15)$ & $0.0051(14)$ & $0.0014(14)$ \\
C13 & $0.0132(16)$ & $0.0105(18)$ & $0.0148(18)$ & $-0.0021(14)$ & $0.0015(14)$ & $0.0005(14)$ \\
C14 & $0.0137(17)$ & $0.0102(19)$ & $0.0181(19)$ & $0.0008(14)$ & $0.0034(14)$ & $-0.0004(14)$ \\
C15 & $0.0097(16)$ & $0.0136(18)$ & $0.022(2)$ & $0.0015(14)$ & $0.0060(15)$ & $0.0028(15)$ \\
C16 & $0.0113(16)$ & $0.0156(18)$ & $0.0192(19)$ & $-0.0009(16)$ & $0.0064(14)$ & $0.0009(16)$ \\
C17 & $0.0166(18)$ & $0.017(2)$ & $0.024(2)$ & $0.0006(17)$ & $0.0023(15)$ & $-0.0001(17)$ \\
C18 & $0.0131(17)$ & $0.021(2)$ & $0.019(2)$ & $-0.0023(16)$ & $-0.0007(15)$ & $0.0005(16)$ \\
C19 & $0.0161(18)$ & $0.0149(19)$ & $0.021(2)$ & $-0.0087(16)$ & $0.0068(15)$ & $-0.0065(16)$ \\
C20 & $0.0149(18)$ & $0.0127(19)$ & $0.026(2)$ & $-0.0039(15)$ & $0.0109(16)$ & $0.0009(16)$ \\
C21 & $0.0098(15)$ & $0.0173(19)$ & $0.0176(18)$ & $0.0006(16)$ & $0.0044(13)$ & $0.0006(16)$ \\
C22 & $0.0104(16)$ & $0.0082(17)$ & $0.020(2)$ & $-0.0028(14)$ & $0.0026(14)$ & $-0.0002(14)$ \\
C23 & $0.0154(18)$ & $0.0137(19)$ & $0.021(2)$ & $0.0025(15)$ & $0.0041(15)$ & $-0.0013(16)$ \\
C24 & $0.0182(19)$ & $0.0120(19)$ & $0.019(2)$ & $0.0019(16)$ & $-0.0017(16)$ & $-0.0011(15)$ \\
C25 & $0.0112(17)$ & $0.0096(18)$ & $0.030(2)$ & $0.0011(15)$ & $-0.0007(16)$ & $-0.0008(16)$ \\
C26 & $0.0128(18)$ & $0.016(2)$ & $0.027(2)$ & $-0.0007(16)$ & $0.0084(16)$ & $-0.0004(17)$ \\
C27 & $0.0150(18)$ & $0.0113(18)$ & $0.018(2)$ & $0.0007(15)$ & $0.0027(15)$ & $-0.0013(15)$ \\
C28 & $0.0177(19)$ & $0.017(2)$ & $0.028(2)$ & $0.0032(16)$ & $0.0070(17)$ & $-0.0049(17)$ \\
& & & & & & \\
\hline
\end{tabular}

Geometric parameters $\left(\AA,{ }^{\circ}\right)$

\begin{tabular}{llll}
\hline $\mathrm{F} 1-\mathrm{C} 19$ & $1.362(4)$ & $\mathrm{C} 13-\mathrm{C} 14$ & $1.400(5)$ \\
$\mathrm{F} 2-\mathrm{C} 25$ & $1.366(4)$ & $\mathrm{C} 13-\mathrm{C} 22$ & $1.499(5)$ \\
$\mathrm{Br} 1-\mathrm{C} 1$ & $1.899(4)$ & $\mathrm{C} 14-\mathrm{C} 15$ & $1.389(5)$ \\
$\mathrm{O} 1-\mathrm{C} 15$ & $1.366(4)$ & $\mathrm{C} 14-\mathrm{H} 14 \mathrm{~A}$ & 0.9300 \\
$\mathrm{O} 1-\mathrm{C} 28$ & $1.432(5)$ & $\mathrm{C} 16-\mathrm{C} 21$ & $1.398(5)$ \\
$\mathrm{O} 2-\mathrm{C} 9$ & $1.227(5)$ & $\mathrm{C} 16-\mathrm{C} 17$ & $1.398(5)$ \\
$\mathrm{C} 1-\mathrm{C} 2$ & $1.382(5)$ & $\mathrm{C} 17-\mathrm{C} 18$ & $1.378(6)$ \\
$\mathrm{C} 1-\mathrm{C} 6$ & $1.405(5)$ & $\mathrm{C} 17-\mathrm{H} 17 \mathrm{~A}$ & 0.9300 \\
$\mathrm{C} 2-\mathrm{C} 3$ & $1.383(6)$ & $\mathrm{C} 18-\mathrm{C} 19$ & $1.377(6)$ \\
$\mathrm{C} 2-\mathrm{H} 2 \mathrm{~A}$ & 0.9300 & $\mathrm{C} 18-\mathrm{H} 18 \mathrm{~A}$ & 0.9300 \\
$\mathrm{C} 3-\mathrm{C} 4$ & $1.380(6)$ & $\mathrm{C} 19-\mathrm{C} 20$ & $1.375(5)$ \\
$\mathrm{C} 3-\mathrm{H} 3 \mathrm{~A}$ & 0.9300 & $\mathrm{C} 20-\mathrm{C} 21$ & $1.386(5)$ \\
$\mathrm{C} 4-\mathrm{C} 5$ & $1.377(6)$ & $\mathrm{C} 20-\mathrm{H} 20 \mathrm{~A}$ & 0.9300 \\
$\mathrm{C} 4-\mathrm{H} 4 \mathrm{~A}$ & 0.9300 & $\mathrm{C} 21-\mathrm{H} 21 \mathrm{~A}$ & 0.9300 \\
$\mathrm{C} 5-\mathrm{C} 6$ & $1.409(6)$ & $\mathrm{C} 22-\mathrm{C} 23$ & $1.392(5)$ \\
$\mathrm{C} 5-\mathrm{H} 5 \mathrm{~A}$ & 0.9300 & $\mathrm{C} 22-\mathrm{C} 27$ & $1.395(5)$ \\
$\mathrm{C} 6-\mathrm{C} 7$ & $1.465(5)$ & $\mathrm{C} 23-\mathrm{C} 24$ & $1.386(5)$ \\
$\mathrm{C} 7-\mathrm{C} 8$ & $1.329(5)$ & $\mathrm{C} 23-\mathrm{H} 23 \mathrm{~A}$ & 0.9300
\end{tabular}




\begin{tabular}{|c|c|c|c|}
\hline C7-H7A & 0.9300 & $\mathrm{C} 24-\mathrm{C} 25$ & $1.384(6)$ \\
\hline $\mathrm{C} 8-\mathrm{C} 9$ & $1.490(5)$ & $\mathrm{C} 24-\mathrm{H} 24 \mathrm{~A}$ & 0.9300 \\
\hline $\mathrm{C} 8-\mathrm{H} 8 \mathrm{~A}$ & 0.9300 & $\mathrm{C} 25-\mathrm{C} 26$ & $1.365(6)$ \\
\hline $\mathrm{C} 9-\mathrm{C} 10$ & $1.503(5)$ & $\mathrm{C} 26-\mathrm{C} 27$ & $1.398(5)$ \\
\hline $\mathrm{C} 10-\mathrm{C} 11$ & $1.403(5)$ & $\mathrm{C} 26-\mathrm{H} 26 \mathrm{~A}$ & 0.9300 \\
\hline $\mathrm{C} 10-\mathrm{C} 15$ & $1.407(5)$ & $\mathrm{C} 27-\mathrm{H} 27 \mathrm{~A}$ & 0.9300 \\
\hline $\mathrm{C} 11-\mathrm{C} 12$ & $1.399(5)$ & $\mathrm{C} 28-\mathrm{H} 28 \mathrm{~A}$ & 0.9600 \\
\hline $\mathrm{C} 11-\mathrm{C} 16$ & $1.483(5)$ & $\mathrm{C} 28-\mathrm{H} 28 \mathrm{~B}$ & 0.9600 \\
\hline $\mathrm{C} 12-\mathrm{C} 13$ & $1.393(5)$ & $\mathrm{C} 28-\mathrm{H} 28 \mathrm{C}$ & 0.9600 \\
\hline $\mathrm{C} 12-\mathrm{H} 12 \mathrm{~A}$ & 0.9300 & & \\
\hline $\mathrm{C} 15-\mathrm{O} 1-\mathrm{C} 28$ & $118.3(3)$ & $\mathrm{O} 1-\mathrm{C} 15-\mathrm{C} 10$ & $115.0(3)$ \\
\hline $\mathrm{C} 2-\mathrm{C} 1-\mathrm{C} 6$ & $122.1(4)$ & $\mathrm{C} 14-\mathrm{C} 15-\mathrm{C} 10$ & $120.8(3)$ \\
\hline $\mathrm{C} 2-\mathrm{C} 1-\mathrm{Br} 1$ & $117.9(3)$ & $\mathrm{C} 21-\mathrm{C} 16-\mathrm{C} 17$ & $117.9(4)$ \\
\hline $\mathrm{C} 6-\mathrm{C} 1-\mathrm{Br} 1$ & $120.0(3)$ & $\mathrm{C} 21-\mathrm{C} 16-\mathrm{C} 11$ & $119.3(3)$ \\
\hline $\mathrm{C} 1-\mathrm{C} 2-\mathrm{C} 3$ & $119.8(4)$ & $\mathrm{C} 17-\mathrm{C} 16-\mathrm{C} 11$ & $122.8(4)$ \\
\hline $\mathrm{C} 1-\mathrm{C} 2-\mathrm{H} 2 \mathrm{~A}$ & 120.1 & $\mathrm{C} 18-\mathrm{C} 17-\mathrm{C} 16$ & $120.9(4)$ \\
\hline $\mathrm{C} 3-\mathrm{C} 2-\mathrm{H} 2 \mathrm{~A}$ & 120.1 & $\mathrm{C} 18-\mathrm{C} 17-\mathrm{H} 17 \mathrm{~A}$ & 119.6 \\
\hline $\mathrm{C} 4-\mathrm{C} 3-\mathrm{C} 2$ & $119.8(4)$ & $\mathrm{C} 16-\mathrm{C} 17-\mathrm{H} 17 \mathrm{~A}$ & 119.6 \\
\hline $\mathrm{C} 4-\mathrm{C} 3-\mathrm{H} 3 \mathrm{~A}$ & 120.1 & $\mathrm{C} 19-\mathrm{C} 18-\mathrm{C} 17$ & $119.2(4)$ \\
\hline $\mathrm{C} 2-\mathrm{C} 3-\mathrm{H} 3 \mathrm{~A}$ & 120.1 & $\mathrm{C} 19-\mathrm{C} 18-\mathrm{H} 18 \mathrm{~A}$ & 120.4 \\
\hline $\mathrm{C} 5-\mathrm{C} 4-\mathrm{C} 3$ & $120.2(4)$ & $\mathrm{C} 17-\mathrm{C} 18-\mathrm{H} 18 \mathrm{~A}$ & 120.4 \\
\hline $\mathrm{C} 5-\mathrm{C} 4-\mathrm{H} 4 \mathrm{~A}$ & 119.9 & $\mathrm{~F} 1-\mathrm{C} 19-\mathrm{C} 20$ & $119.2(4)$ \\
\hline $\mathrm{C} 3-\mathrm{C} 4-\mathrm{H} 4 \mathrm{~A}$ & 119.9 & $\mathrm{~F} 1-\mathrm{C} 19-\mathrm{C} 18$ & $118.7(3)$ \\
\hline $\mathrm{C} 4-\mathrm{C} 5-\mathrm{C} 6$ & $122.0(4)$ & $\mathrm{C} 20-\mathrm{C} 19-\mathrm{C} 18$ & $122.1(4)$ \\
\hline $\mathrm{C} 4-\mathrm{C} 5-\mathrm{H} 5 \mathrm{~A}$ & 119.0 & $\mathrm{C} 19-\mathrm{C} 20-\mathrm{C} 21$ & $118.0(4)$ \\
\hline $\mathrm{C} 6-\mathrm{C} 5-\mathrm{H} 5 \mathrm{~A}$ & 119.0 & $\mathrm{C} 19-\mathrm{C} 20-\mathrm{H} 20 \mathrm{~A}$ & 121.0 \\
\hline $\mathrm{C} 1-\mathrm{C} 6-\mathrm{C} 5$ & $116.0(3)$ & $\mathrm{C} 21-\mathrm{C} 20-\mathrm{H} 20 \mathrm{~A}$ & 121.0 \\
\hline $\mathrm{C} 1-\mathrm{C} 6-\mathrm{C} 7$ & $122.1(4)$ & $\mathrm{C} 20-\mathrm{C} 21-\mathrm{C} 16$ & $121.8(3)$ \\
\hline $\mathrm{C} 5-\mathrm{C} 6-\mathrm{C} 7$ & $121.9(3)$ & $\mathrm{C} 20-\mathrm{C} 21-\mathrm{H} 21 \mathrm{~A}$ & 119.1 \\
\hline $\mathrm{C} 8-\mathrm{C} 7-\mathrm{C} 6$ & $126.1(4)$ & $\mathrm{C} 16-\mathrm{C} 21-\mathrm{H} 21 \mathrm{~A}$ & 119.1 \\
\hline $\mathrm{C} 8-\mathrm{C} 7-\mathrm{H} 7 \mathrm{~A}$ & 116.9 & $\mathrm{C} 23-\mathrm{C} 22-\mathrm{C} 27$ & $119.7(3)$ \\
\hline $\mathrm{C} 6-\mathrm{C} 7-\mathrm{H} 7 \mathrm{~A}$ & 116.9 & $\mathrm{C} 23-\mathrm{C} 22-\mathrm{C} 13$ & $119.4(3)$ \\
\hline $\mathrm{C} 7-\mathrm{C} 8-\mathrm{C} 9$ & $122.0(4)$ & $\mathrm{C} 27-\mathrm{C} 22-\mathrm{C} 13$ & $120.8(3)$ \\
\hline $\mathrm{C} 7-\mathrm{C} 8-\mathrm{H} 8 \mathrm{~A}$ & 119.0 & $\mathrm{C} 24-\mathrm{C} 23-\mathrm{C} 22$ & $120.3(4)$ \\
\hline $\mathrm{C} 9-\mathrm{C} 8-\mathrm{H} 8 \mathrm{~A}$ & 119.0 & $\mathrm{C} 24-\mathrm{C} 23-\mathrm{H} 23 \mathrm{~A}$ & 119.9 \\
\hline $\mathrm{O} 2-\mathrm{C} 9-\mathrm{C} 8$ & $122.1(3)$ & $\mathrm{C} 22-\mathrm{C} 23-\mathrm{H} 23 \mathrm{~A}$ & 119.9 \\
\hline $\mathrm{O} 2-\mathrm{C} 9-\mathrm{C} 10$ & $120.9(3)$ & $\mathrm{C} 25-\mathrm{C} 24-\mathrm{C} 23$ & $118.5(4)$ \\
\hline $\mathrm{C} 8-\mathrm{C} 9-\mathrm{C} 10$ & $117.0(3)$ & $\mathrm{C} 25-\mathrm{C} 24-\mathrm{H} 24 \mathrm{~A}$ & 120.8 \\
\hline $\mathrm{C} 11-\mathrm{C} 10-\mathrm{C} 15$ & $119.7(3)$ & $\mathrm{C} 23-\mathrm{C} 24-\mathrm{H} 24 \mathrm{~A}$ & 120.8 \\
\hline $\mathrm{C} 11-\mathrm{C} 10-\mathrm{C} 9$ & $122.6(3)$ & $\mathrm{C} 26-\mathrm{C} 25-\mathrm{F} 2$ & $119.0(3)$ \\
\hline $\mathrm{C} 15-\mathrm{C} 10-\mathrm{C} 9$ & $117.7(3)$ & $\mathrm{C} 26-\mathrm{C} 25-\mathrm{C} 24$ & $122.9(4)$ \\
\hline $\mathrm{C} 12-\mathrm{C} 11-\mathrm{C} 10$ & $118.6(3)$ & $\mathrm{F} 2-\mathrm{C} 25-\mathrm{C} 24$ & $118.1(3)$ \\
\hline $\mathrm{C} 12-\mathrm{C} 11-\mathrm{C} 16$ & $119.4(3)$ & $\mathrm{C} 25-\mathrm{C} 26-\mathrm{C} 27$ & $118.4(4)$ \\
\hline $\mathrm{C} 10-\mathrm{C} 11-\mathrm{C} 16$ & $122.0(3)$ & $\mathrm{C} 25-\mathrm{C} 26-\mathrm{H} 26 \mathrm{~A}$ & 120.8 \\
\hline $\mathrm{C} 13-\mathrm{C} 12-\mathrm{C} 11$ & $121.8(3)$ & $\mathrm{C} 27-\mathrm{C} 26-\mathrm{H} 26 \mathrm{~A}$ & 120.8 \\
\hline $\mathrm{C} 13-\mathrm{C} 12-\mathrm{H} 12 \mathrm{~A}$ & 119.1 & $\mathrm{C} 22-\mathrm{C} 27-\mathrm{C} 26$ & $120.1(4)$ \\
\hline $\mathrm{C} 11-\mathrm{C} 12-\mathrm{H} 12 \mathrm{~A}$ & 119.1 & $\mathrm{C} 22-\mathrm{C} 27-\mathrm{H} 27 \mathrm{~A}$ & 119.9 \\
\hline
\end{tabular}




\begin{tabular}{|c|c|}
\hline $\mathrm{C} 12-\mathrm{C} 13-\mathrm{C} 14$ & $119.2(3)$ \\
\hline $\mathrm{C} 12-\mathrm{C} 13-\mathrm{C} 22$ & $120.9(3)$ \\
\hline $\mathrm{C} 14-\mathrm{C} 13-\mathrm{C} 22$ & $119.8(3)$ \\
\hline $\mathrm{C} 15-\mathrm{C} 14-\mathrm{C} 13$ & $119.9(3)$ \\
\hline $\mathrm{C} 15-\mathrm{C} 14-\mathrm{H} 14 \mathrm{~A}$ & 120.1 \\
\hline $\mathrm{C} 13-\mathrm{C} 14-\mathrm{H} 14 \mathrm{~A}$ & 120.1 \\
\hline $\mathrm{O} 1-\mathrm{C} 15-\mathrm{C} 14$ & $124.2(3)$ \\
\hline $\mathrm{C} 6-\mathrm{C} 1-\mathrm{C} 2-\mathrm{C} 3$ & $0.4(6)$ \\
\hline $\mathrm{Br} 1-\mathrm{C} 1-\mathrm{C} 2-\mathrm{C} 3$ & $179.7(3)$ \\
\hline $\mathrm{C} 1-\mathrm{C} 2-\mathrm{C} 3-\mathrm{C} 4$ & $1.0(6)$ \\
\hline $\mathrm{C} 2-\mathrm{C} 3-\mathrm{C} 4-\mathrm{C} 5$ & $-0.4(6)$ \\
\hline $\mathrm{C} 3-\mathrm{C} 4-\mathrm{C} 5-\mathrm{C} 6$ & $-1.7(7)$ \\
\hline $\mathrm{C} 2-\mathrm{C} 1-\mathrm{C} 6-\mathrm{C} 5$ & $-2.4(6)$ \\
\hline $\mathrm{Br} 1-\mathrm{C} 1-\mathrm{C} 6-\mathrm{C} 5$ & $178.4(3)$ \\
\hline $\mathrm{C} 2-\mathrm{C} 1-\mathrm{C} 6-\mathrm{C} 7$ & $177.6(4)$ \\
\hline $\mathrm{Br} 1-\mathrm{C} 1-\mathrm{C} 6-\mathrm{C} 7$ & $-1.7(5)$ \\
\hline $\mathrm{C} 4-\mathrm{C} 5-\mathrm{C} 6-\mathrm{C} 1$ & $3.0(6)$ \\
\hline $\mathrm{C} 4-\mathrm{C} 5-\mathrm{C} 6-\mathrm{C} 7$ & $-176.9(4)$ \\
\hline $\mathrm{C} 1-\mathrm{C} 6-\mathrm{C} 7-\mathrm{C} 8$ & $178.2(4)$ \\
\hline $\mathrm{C} 5-\mathrm{C} 6-\mathrm{C} 7-\mathrm{C} 8$ & $-1.9(7)$ \\
\hline $\mathrm{C} 6-\mathrm{C} 7-\mathrm{C} 8-\mathrm{C} 9$ & $178.5(4)$ \\
\hline $\mathrm{C} 7-\mathrm{C} 8-\mathrm{C} 9-\mathrm{O} 2$ & $-2.9(6)$ \\
\hline $\mathrm{C} 7-\mathrm{C} 8-\mathrm{C} 9-\mathrm{C} 10$ & $176.8(4)$ \\
\hline $\mathrm{O} 2-\mathrm{C} 9-\mathrm{C} 10-\mathrm{C} 11$ & $-55.4(5)$ \\
\hline $\mathrm{C} 8-\mathrm{C} 9-\mathrm{C} 10-\mathrm{C} 11$ & $124.8(4)$ \\
\hline $\mathrm{O} 2-\mathrm{C} 9-\mathrm{C} 10-\mathrm{C} 15$ & $122.9(4)$ \\
\hline $\mathrm{C} 8-\mathrm{C} 9-\mathrm{C} 10-\mathrm{C} 15$ & $-56.9(5)$ \\
\hline $\mathrm{C} 15-\mathrm{C} 10-\mathrm{C} 11-\mathrm{C} 12$ & $-0.6(5)$ \\
\hline $\mathrm{C} 9-\mathrm{C} 10-\mathrm{C} 11-\mathrm{C} 12$ & $177.7(3)$ \\
\hline $\mathrm{C} 15-\mathrm{C} 10-\mathrm{C} 11-\mathrm{C} 16$ & $179.0(3)$ \\
\hline $\mathrm{C} 9-\mathrm{C} 10-\mathrm{C} 11-\mathrm{C} 16$ & $-2.7(6)$ \\
\hline $\mathrm{C} 10-\mathrm{C} 11-\mathrm{C} 12-\mathrm{C} 13$ & $-1.2(5)$ \\
\hline $\mathrm{C} 16-\mathrm{C} 11-\mathrm{C} 12-\mathrm{C} 13$ & $179.2(3)$ \\
\hline $\mathrm{C} 11-\mathrm{C} 12-\mathrm{C} 13-\mathrm{C} 14$ & $1.7(5)$ \\
\hline $\mathrm{C} 11-\mathrm{C} 12-\mathrm{C} 13-\mathrm{C} 22$ & $-176.2(3)$ \\
\hline $\mathrm{C} 12-\mathrm{C} 13-\mathrm{C} 14-\mathrm{C} 15$ & $-0.3(5)$ \\
\hline $\mathrm{C} 22-\mathrm{C} 13-\mathrm{C} 14-\mathrm{C} 15$ & $177.6(3)$ \\
\hline $\mathrm{C} 28-\mathrm{O} 1-\mathrm{C} 15-\mathrm{C} 14$ & $-12.1(5)$ \\
\hline $\mathrm{C} 28-\mathrm{O} 1-\mathrm{C} 15-\mathrm{C} 10$ & $169.7(3)$ \\
\hline $\mathrm{C} 13-\mathrm{C} 14-\mathrm{C} 15-\mathrm{O} 1$ & $-179.6(3)$ \\
\hline
\end{tabular}

\begin{tabular}{|c|c|}
\hline $\mathrm{C} 26-\mathrm{C} 27-\mathrm{H} 27 \mathrm{~A}$ & 119.9 \\
\hline $\mathrm{O} 1-\mathrm{C} 28-\mathrm{H} 28 \mathrm{~A}$ & 109.5 \\
\hline $\mathrm{O} 1-\mathrm{C} 28-\mathrm{H} 28 \mathrm{~B}$ & 109.5 \\
\hline $\mathrm{H} 28 \mathrm{~A}-\mathrm{C} 28-\mathrm{H} 28 \mathrm{~B}$ & 109.5 \\
\hline $\mathrm{O} 1-\mathrm{C} 28-\mathrm{H} 28 \mathrm{C}$ & 109.5 \\
\hline $\mathrm{H} 28 \mathrm{~A}-\mathrm{C} 28-\mathrm{H} 28 \mathrm{C}$ & 109.5 \\
\hline $\mathrm{H} 28 \mathrm{~B}-\mathrm{C} 28-\mathrm{H} 28 \mathrm{C}$ & 109.5 \\
\hline $\mathrm{C} 13-\mathrm{C} 14-\mathrm{C} 15-\mathrm{C} 10$ & $-1.5(6)$ \\
\hline $\mathrm{C} 11-\mathrm{C} 10-\mathrm{C} 15-\mathrm{O} 1$ & $-179.8(3)$ \\
\hline $\mathrm{C} 9-\mathrm{C} 10-\mathrm{C} 15-\mathrm{O} 1$ & $1.8(5)$ \\
\hline $\mathrm{C} 11-\mathrm{C} 10-\mathrm{C} 15-\mathrm{C} 14$ & $1.9(6)$ \\
\hline $\mathrm{C} 9-\mathrm{C} 10-\mathrm{C} 15-\mathrm{C} 14$ & $-176.4(3)$ \\
\hline $\mathrm{C} 12-\mathrm{C} 11-\mathrm{C} 16-\mathrm{C} 21$ & $-47.1(5)$ \\
\hline $\mathrm{C} 10-\mathrm{C} 11-\mathrm{C} 16-\mathrm{C} 21$ & $133.3(4)$ \\
\hline $\mathrm{C} 12-\mathrm{C} 11-\mathrm{C} 16-\mathrm{C} 17$ & $133.3(4)$ \\
\hline $\mathrm{C} 10-\mathrm{C} 11-\mathrm{C} 16-\mathrm{C} 17$ & $-46.2(5)$ \\
\hline $\mathrm{C} 21-\mathrm{C} 16-\mathrm{C} 17-\mathrm{C} 18$ & $-2.1(6)$ \\
\hline $\mathrm{C} 11-\mathrm{C} 16-\mathrm{C} 17-\mathrm{C} 18$ & $177.5(4)$ \\
\hline $\mathrm{C} 16-\mathrm{C} 17-\mathrm{C} 18-\mathrm{C} 19$ & $-0.4(6)$ \\
\hline $\mathrm{C} 17-\mathrm{C} 18-\mathrm{C} 19-\mathrm{F} 1$ & $-177.1(3)$ \\
\hline $\mathrm{C} 17-\mathrm{C} 18-\mathrm{C} 19-\mathrm{C} 20$ & $3.5(6)$ \\
\hline $\mathrm{F} 1-\mathrm{C} 19-\mathrm{C} 20-\mathrm{C} 21$ & $176.6(3)$ \\
\hline $\mathrm{C} 18-\mathrm{C} 19-\mathrm{C} 20-\mathrm{C} 21$ & $-4.0(6)$ \\
\hline $\mathrm{C} 19-\mathrm{C} 20-\mathrm{C} 21-\mathrm{C} 16$ & $1.3(5)$ \\
\hline $\mathrm{C} 17-\mathrm{C} 16-\mathrm{C} 21-\mathrm{C} 20$ & $1.6(5)$ \\
\hline $\mathrm{C} 11-\mathrm{C} 16-\mathrm{C} 21-\mathrm{C} 20$ & $-178.0(3)$ \\
\hline $\mathrm{C} 12-\mathrm{C} 13-\mathrm{C} 22-\mathrm{C} 23$ & $130.6(4)$ \\
\hline $\mathrm{C} 14-\mathrm{C} 13-\mathrm{C} 22-\mathrm{C} 23$ & $-47.2(5)$ \\
\hline $\mathrm{C} 12-\mathrm{C} 13-\mathrm{C} 22-\mathrm{C} 27$ & $-47.8(5)$ \\
\hline $\mathrm{C} 14-\mathrm{C} 13-\mathrm{C} 22-\mathrm{C} 27$ & $134.4(4)$ \\
\hline $\mathrm{C} 27-\mathrm{C} 22-\mathrm{C} 23-\mathrm{C} 24$ & $0.2(6)$ \\
\hline $\mathrm{C} 13-\mathrm{C} 22-\mathrm{C} 23-\mathrm{C} 24$ & $-178.2(3)$ \\
\hline $\mathrm{C} 22-\mathrm{C} 23-\mathrm{C} 24-\mathrm{C} 25$ & $-0.7(6)$ \\
\hline $\mathrm{C} 23-\mathrm{C} 24-\mathrm{C} 25-\mathrm{C} 26$ & $0.1(6)$ \\
\hline $\mathrm{C} 23-\mathrm{C} 24-\mathrm{C} 25-\mathrm{F} 2$ & $-179.6(3)$ \\
\hline $\mathrm{F} 2-\mathrm{C} 25-\mathrm{C} 26-\mathrm{C} 27$ & $-179.4(3)$ \\
\hline $\mathrm{C} 24-\mathrm{C} 25-\mathrm{C} 26-\mathrm{C} 27$ & $0.8(6)$ \\
\hline $\mathrm{C} 23-\mathrm{C} 22-\mathrm{C} 27-\mathrm{C} 26$ & $0.7(6)$ \\
\hline $\mathrm{C} 13-\mathrm{C} 22-\mathrm{C} 27-\mathrm{C} 26$ & $179.1(3)$ \\
\hline $\mathrm{C} 25-\mathrm{C} 26-\mathrm{C} 27-\mathrm{C} 22$ & $-1.2(6)$ \\
\hline
\end{tabular}

Hydrogen-bond geometry $\left(\AA,{ }^{\circ}\right)$

$\mathrm{Cg} 1$ and $\mathrm{Cg} 2$ are the centroids of $\mathrm{C} 1-\mathrm{C} 6$ and $\mathrm{C} 10-\mathrm{C} 15$ rings, respectively.

\begin{tabular}{lllll}
\hline$D-\mathrm{H} \cdots A$ & $D-\mathrm{H}$ & $\mathrm{H} \cdots A$ & $D \cdots A$ & $D-\mathrm{H} \cdots A$ \\
\hline $\mathrm{C} 28-\mathrm{H} 28 A \cdots \mathrm{F} 2^{\mathrm{i}}$ & 0.96 & 2.51 & $3.448(4)$ & 166 \\
$\mathrm{C} 4-\mathrm{H} 4 A \cdots C g 1^{\mathrm{ii}}$ & 0.93 & 2.99 & $3.712(5)$ & 136
\end{tabular}


supporting information

\begin{tabular}{lllll}
$\mathrm{C} 20-\mathrm{H} 20 A \cdots C g 2^{\mathrm{iii}}$ & 0.93 & 2.72 & $3.383(4)$ & 129 \\
$\mathrm{C} 27-\mathrm{H} 27 A \cdots C g 1^{\mathrm{iv}}$ & 0.93 & 2.95 & $3.735(4)$ & 143 \\
$\mathrm{C} 28-\mathrm{H} 28 B \cdots C g 2^{\mathrm{v}}$ & 0.96 & 2.82 & $3.485(4)$ & 128 \\
\hline
\end{tabular}

Symmetry codes: (i) $x+1 / 2, y-1 / 2, z$; (ii) $x,-y-1, z-1 / 2$; (iii) $x, y+1, z$; (iv) $-x-1,-y,-z$; (v) $x+1 / 2, y+3 / 2, z$. 